\title{
ELLIPTIC CALABI-YAU THREEFOLDS OVER A DEL PEZZO SURFACE
}

\author{
SIMON ROSE AND NORIKO YUI
}

\begin{abstract}
We consider certain elliptic threefolds over the projective plane (more generally over certain rational surfaces) with a section in Weierstrass normal form. In particular, over a del Pezzo surface of degree 8, these elliptic threefolds are Calabi-Yau threefolds. We will discuss especially the generating functions of Gromov-Witten and Gopakumar-Vafa invariants.
\end{abstract}

\section{INTRODUCTION}

During a visit to Max-Planck-Institut für Mathematik Bonn in the spring of 2004, Professor Hirzebruch showed the second author a specific construction of CalabiYau threefolds, which are elliptic threefolds over a del Pezzo surface of degree 8 in Weierstrass normal form, that is a family of elliptic curves over a del Pezzo surface of degree 8 (a rational surface) [5]. The purpose of this short note is to discuss the generating functions of Gromov-Witten and Gopakumar-Vafa invariants.

This paper was completed while both authors were in residence at the Fields Institute for the thematic program. We thank the hospitality of the Fields Institute.

\section{A Del Pezzo Surface of Degree 8}

First we will give a definition of a del Pezzo surface. A good reference is Manin [11.

Definition 2.1. A del Pezzo surface $S$ is a smooth projective geometrically irreducible surface whose anti-canonical bundle is ample, i.e., $-K_{S}$ is ample.

The degree of $S$ is a positive integer defined by

$$
\operatorname{deg} S:=K_{S} \cdot K_{S} .
$$

That is, the degree of $S$ is the self-intersection of its canonical class.

Date: October 2, 2018.

2000 Mathematics Subject Classification. Primary ;14N10, 11F11, 14 H52.

Key words and phrases. del Pezzo surface, Calabi-Yau threefold, Modular Forms.

S. Rose was supported by a Jerry Marsden Postdoctoral Fellowship for the Fields major thematic program on Calabi-Yau Varieties: Arithmetic, Geometry and Physics from July to December 2013.

N. Yui was supported in part by the Natural Sciences and Engineering Research Council (NSERC) Discovery Grant. 
Remark 2.1. (1) Every del Pezzo surface is geometrically rational. Therefore, it is birationally equivalent to the projective plane, $\mathbb{P}^{2}$.

(2) Let $S$ be a del Pezzo surface. Then $1 \leq \operatorname{deg} S \leq 9$.

(3) If $\operatorname{deg} S>2$, then its anti-canonical bundle $-K_{S}$ is very ample.

Here is a classification results of del Pezzo surfaces according to their degrees.

Theorem 2.1. Let $S$ be a del Pezzo surface.

(a) If $\operatorname{deg} S=4, S$ is birationally equivalent to a complete intersection of two quadrics in $\mathbb{P}^{4}$.

(b) If $\operatorname{deg} S=3, S$ is birationally equivalent to a cubic surface in $\mathbb{P}^{3}$.

(c) If degS $=2, S$ is birationally equivalent to a hypersurface of degree 4 in the weighted projective 2 -space $\mathbb{P}(2,1,1,1)$.

(d) If deg $S=1, S$ is birationally equivalent to a hypersurface of degree 6 in the weighted projective 2 -space $\mathbb{P}(3,2,1,1)$.

(e) Any smooth surface as in (a),(b),(c) or (d) is del Pezzo surface of the expected degree.

(f) Let $P_{1}, P_{2}, \cdots, P_{r}$ with $r \leq 8$ be generic points in $\mathbb{P}^{2}$. Let $S:=B r_{P_{1}, \cdots, P_{r}}\left(\mathbb{P}^{2}\right)$ be the blow-up of $\mathbb{P}^{2}$ at $P_{i}, 1 \leq i \leq r$. Then $S$ is a del Pezzo surface of degree $9-r$.

To obtain a del Pezzo surface of degree 8, we blow-up $\mathbb{P}^{2}$ in one point.

Corollary 2.2. Pick a point $P \in \mathbb{P}^{2}$, and a line $H \subset \mathbb{P}^{2}$ not passing through $P$. Then

$$
-K_{\mathbb{P}^{2}}=3 H, \quad \text { and } \quad K_{\mathbb{P}^{2}} \cdot K_{\mathbb{P}^{2}}=9 H^{2}=9 .
$$

Let $S:=B l_{P}\left(\mathbb{P}^{2}\right)$ be the blow-up of $\mathbb{P}^{2}$ at $P$. Furthermore, let $E$ denote the exceptional curve replacing $P$; then $E \cdot E=-1$. Let $\xi: S \rightarrow \mathbb{P}^{2}$ be the blow-up map. Then

$$
K_{S}=\xi^{*}\left(K_{\mathbb{P}^{2}}\right)+E
$$

and

$$
\begin{aligned}
K_{S} \cdot K_{S} & =\xi^{*}\left(K_{\mathbb{P}^{2}}\right) \cdot \xi^{*}\left(K_{\mathbb{P}^{2}}\right)+2 \xi^{*}\left(K_{\mathbb{P}^{2}}\right) \cdot E+E \cdot E \\
& =K_{\mathbb{P}^{2}} \cdot K_{\mathbb{P}^{2}}+E \cdot E \\
& =9-1=8 .
\end{aligned}
$$

Then $S$ is a del Pezzo surface of degree 8.

Remark 2.2. Let $S$ be a del Pezzo surface of degree $d$. Then

(1) Every irreducible curve on $S$ is exceptional.

(2) If $S$ has no exceptional curves, then either $d=9$ and $S$ is isomorphic to $\mathbb{P}^{2}$, or $d=8$ and $S$ is isomorphic to $\mathbb{P}^{1} \times \mathbb{P}^{1}$.

(3) If $S$ is not isomorphic to $\mathbb{P}^{1} \times \mathbb{P}^{1}$, then the Picard group Pic(S) is isomorphic to $\mathbb{Z}^{10-d}$. In particular, if $d=8, \operatorname{Pic}(S) \simeq \mathbb{Z}^{2}$ and is spanned by $H$ and $E$.

\section{The Construction of Elliptic threefolds over $S$}

Let $\pi: X \rightarrow S$ be an elliptic fibration, and let $L$ be a line bundle on $S$ with $L \cdot L=8$. Take

i.e.,

$$
g_{2} \in H^{0}\left(S, L^{4}\right), \text { and } g_{3} \in H^{0}\left(S, L^{6}\right),
$$

$$
g_{2}=4 L \quad \text { and } \quad g_{3}=6 L
$$


and let

$$
X: y^{2} z=4 x^{3}-g_{2} x z^{2}-g_{3} z^{3} .
$$

Then the canonial bundle $K_{X}$ is given by

$$
K_{X}=\pi^{*}\left(K_{X / S}+K_{S}\right) \quad \text { with } K_{X / S} \simeq L^{-1} .
$$

We want $X$ to be a Calabi-Yau threefold. The Calabi-Yau condition imposes that

$$
K_{X} \simeq \mathcal{O}_{X} \Longleftrightarrow K_{S}=L^{-1} \Longleftrightarrow-K_{S}=L .
$$

Now

$$
K_{S}=-3 H+E \Longleftrightarrow L=3 H-E
$$

so that

$$
4 L=4(3 H-E) \quad \text { and } \quad 6 L=6(3 H-E) .
$$

Let $\left[z_{0}: z_{1}: z_{2}\right]$ be the projective coordinate for $\mathbb{P}^{2}$. Then $g_{2}=g_{2}\left(z_{0}, z_{1}, z_{2}\right) \in 4 L$ and is of degree 12. While $g_{3}=g_{3}\left(z_{0}, z_{1}, z_{2}\right) \in 6 L$ and is of degree 18. Put $\Delta=4 g_{2}^{3}-27 g_{3}^{2}$. Then $\Delta=\Delta\left(z_{0}, z_{1}, z_{2}\right) \in 12 L$ and is of degree 36 .

\section{Calculation of the Euler characteristic and the Hodge numbers}

Let $X$ be an elliptic threefold constructed above. Then by the construction, the geometric genus of $X$ is $p_{g}(X)=1$ and $h^{1,0}(X)=h^{2,0}(X)=0$. So $X$ is a Calabi-Yau threefold. Now we calculate the Euler characteristic $e(X)$ of $X$.

Lemma 4.1. Let $Y$ be a complex surface (possibly with singularities). Then the Euler characteristic $e(Y)(D)$ for any divisor $D$ is given by

$$
e(Y)(D)=K_{Y} \cdot D-D \cdot D+\text { Contribution from singularities. }
$$

In particular, if $Y$ is smooth,

$$
e(Y)(D)=2-2 g(Y)
$$

which is independent of a choice of a divisor $D$.

Proposition 4.2. Let $X: y^{2} z=4 x^{3}-g_{2} x z^{2}-g_{3} z^{3}$ be a Calabi-Yau threefold over a del Pezzo surface $S$, and let $\Delta=4 g_{2}^{3}-27 g_{3}^{2}$. Then the Euler characteristic $e(X)$ of $X$ is given by the formula

$$
e(X)=e(\Delta)+\# \text { cusps }
$$

where the Euler characteristic $e(\Delta)$ of $\{\Delta=0\}$ is given by

$$
e(\Delta)=-\operatorname{deg} K_{\Delta}=2-2 g(\Delta)+2 \# \text { cusps }
$$

where $g(\Delta)$ denotes the genus of $\{\Delta=0\}$.

Moreover, we can compute that

$$
\text { \#cusps }=192 \text { and } g(\Delta)=595 .
$$

Finally, we obtain

$$
e(X)=-480 .
$$


Proof. First recall that $L=-K_{S}$ and that $L \cdot L=8$. Then we have

$$
\begin{gathered}
K_{\Delta}=\left(K_{S}+\Delta\right) \cdot \Delta=(-L+12 L) \cdot 12 L \\
=11 L \cdot 12 L=(11 \cdot 12)(L \cdot L)=11 \cdot 12 \cdot 8=1056 .
\end{gathered}
$$

The number of cusps is given by

$$
4 L \cdot 6 L=24(L \cdot L)=24 \cdot 8=192 .
$$

Then

$$
e(\Delta)=-1056+2 \cdot 192=-1056+384=-672 .
$$

Now we need to calculate the Euler characteristic of resolutions of singularities. If $\{\Delta=0\}$ is smooth, its resolution is an elliptic curve $E$, and the Euler characteristic $e(E)=0$. If $\{\Delta=0\}$ is a node, the Euler characteristic of its resolution is 1 , and if $\{\Delta=0\}$ is a cusp, the Euler characteristic of its resolution is 2 .

Then we have

$$
\begin{gathered}
e(X)=\left(\begin{array}{c}
e(E) \times e\left(\mathbb{P}^{2} \backslash \Delta\right) \\
+e(\Delta \backslash\{\text { cusps }\}) \times e(\text { resolution of a node }) \\
+\# \text { cusps } \times e(\text { resolution of a cusp })
\end{array}\right) \\
=e(\Delta)-\# \text { cusps }+2 \# \text { cusps }=e(\Delta)+\# \text { cusps. }
\end{gathered}
$$

Finally we obtain

$$
e(X)=-672+192=-480 .
$$

The Hodge nubmers $h^{1,1}(X)$ and $h^{2,1}(X)$ have been calculated by Hulek and Kloosterman [6] (Section 11). This is done by calculating the Mordell-Weil rank of the elliptic curve $\pi: X \rightarrow S$, which turns out to be 0 .

\section{Lemma 4.3.}

$$
h^{1,1}(X)=3, \quad \text { and } \quad h^{2,1}(X)=243 .
$$

The topological Euler characteristic is $e(X)=-480$.

Thus the Hodge diamond is given by

$$
\begin{aligned}
& B_{0}(X)=1 \\
& \begin{array}{llll}
0 & 0 & B_{1}(X)=0 \\
0 & 3 & 0 & B_{2}(X)=3
\end{array} \\
& \begin{array}{lllll}
1 & 243 & 243 & 1 & B_{3}(X)
\end{array} \\
& \begin{array}{llll}
0 & 3 & 0 & B_{4}(X)=3
\end{array} \\
& \begin{array}{lll}
0 & 0 & B_{5}(X)=0
\end{array} \\
& 1 \quad B_{6}(X)=1
\end{aligned}
$$

Recall $X$ is defined by a Weierstrass equation over the del Pezzo surface $S$ of degree 8 which is birational to $\mathbb{P}^{2}$,

$$
y^{2} z=4 x^{3}-g_{2} x z^{2}-g_{3} z^{3} \quad \text { where } g_{2}, g_{3} \in \mathbb{C}(S)
$$

the $j$-invariant of $X$ is defined by

$$
j=1728 \frac{g_{2}^{3}}{\Delta} \quad \text { where } \quad \Delta=4 g_{2}^{3}-27 g_{3}^{2} .
$$

As for these elliptic threefolds, we have

Lemma 4.4. The $j$-invariant is a moduli for $X$. 
We are also interested in the modularity question for the Galois representation associated to $X$. However, the Betti number $B_{3}(X)=488$ is too large to make this practical. Thus, we are interested in constructing a topological mirror Calabi-Yau threefold $\check{X}$.

For a topological mirror partner $\check{X}$ of our elliptic Calabi-Yau threefold $X$, the Hodge numbers are

$$
h^{1,1}(\check{X})=243, h^{2,1}(\check{X})=3
$$

and the Euler characteristic is

$$
e(\check{X})=480 \text {. }
$$

The Betti numbers are

$$
B_{2}(\check{X})=243, B_{3}(\check{X})=8 \text {. }
$$

In this case, the modularity of the Galois representation may at least somewhat be tractable. This leads us to ask: How can we construct such a mirror Calabi-Yau threefold?

\section{Gromov-Witten and Gopakumar-Vafa invariants}

We are naturally interested in the Gromov-Witten invariants of the threefold $X$. These are obtained via integration against the virtual fundamental class of the moduli space of stable maps into $X$. That is, we define

$$
N_{g, \beta}^{X}=\int_{\left[M_{g, n}(X, \beta)\right]^{v i r}} 1 .
$$

In the best of cases, these invariants are positive integers and count the number of curves in $X$ in the homology class $\beta$. In many cases, however, since $M_{g, n}(X, \beta)$ is a stack, the invariants are only rational numbers.

Naturally, we organize these invariants into a generating function as follows. Let $F_{g}^{X}(q)$ and $F^{X}(q, \lambda)$ be defined as

$$
\begin{aligned}
& F_{g}^{X}(q)=\sum_{\beta \in H_{2}(X)} N_{g, \beta}^{X} q^{\beta} \\
& F^{X}(q)=\sum_{g=0}^{\infty} \lambda^{2 g-2} F_{g}(q) .
\end{aligned}
$$

We can now define the Gopakumar-Vafa/BPS invariants via the equality

$$
F^{X}(q)=\sum_{g=0}^{\infty} \sum_{\beta \in H_{2}(X)} n_{g, \beta}^{X} \sum_{m=1}^{\infty} \frac{1}{k}\left(2 \sin \left(\frac{k \lambda}{2}\right)\right)^{2 g-2} q^{k \beta} .
$$

For example, the $g=0$ portion of this reads

$$
N_{0, \beta}^{X}=\sum_{\substack{\eta \in H_{2}(X) \\ k \eta=\beta}} \frac{1}{k^{3}} n_{0, \eta}^{X} .
$$

These invariants $n_{g, \beta}^{X}$ are defined recursively in terms of the Gromov-Witten invariants $N_{g, \beta}^{X}$, and a priori these are only rational numbers. It is a conjecture (see $[2,7])$ that they are integers for all $X, g, \beta$. We work with them because in the case of the Calabi-Yau threefold $X$, the formulæ for them turn out to be much simpler; the Gromov-Witten invariants can then be reconstructed from them.

In the case that a class $\beta$ is primitive, the invariants $N_{0, \beta}^{X}$ and $n_{0, \beta}^{X}$ coincide. 


\section{The GeOMEtRy of $X$}

In order to compute these invariants, we need a bit more of a description of the geometry of the 3 -fold $X$. We begin with the following fact. The del Pezzo surface $S$ is in fact isomorphic to the Hirzebruch surface $\mathbb{F}_{1}=\mathbb{P}(\mathcal{O} \oplus \mathcal{O}(1))$. This is a $\mathbb{P}^{1}$ bundle over the base $\mathbb{P}^{1}$, with a $(-1)$-curve as a section. Let $C^{\prime}, F^{\prime}$ denote the homology classes in $S$ of the section and fibre, respectively.

Consider now the following composition

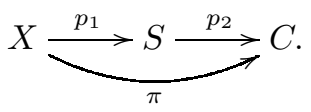

The generic fibre of this is an elliptically fibred K3 surface with $24 I_{1}$ fibres.

Let now $X_{F}$ denote one such generic fibre, and let $X_{C}$ denote the restriction of $X$ to the section $C$. This latter surface is a rational elliptic surface with $12 I_{1}$ fibres (which physicists call a $\frac{1}{2} \mathrm{~K} 3$ ). Similarly, let $C^{\prime \prime}, E^{\prime \prime}$ denote the class of the section and fibre in $X_{C}$, respectively.

We want to have a description of the Picard group and a basis of $H_{2}(X, \mathbb{Z})$. So consider first the line bundles

$$
L_{1}=\mathcal{O}(S) \quad L_{2}=\mathcal{O}\left(X_{C}\right) \quad L_{3}=\mathcal{O}\left(X_{F}\right)
$$

and let $\iota_{1}, \iota_{2}, \iota_{3}$ denote the respective inclusions of $S, X_{C}, X_{F}$. We now define the homology classes

$$
\begin{gathered}
C=\left(\iota_{1}\right)_{*}\left(C^{\prime}\right)=\left(\iota_{2}\right)_{*}\left(C^{\prime \prime}\right) \\
E=\left(\iota_{2}\right)_{*}\left(E^{\prime \prime}\right) \\
F=\left(\iota_{1}\right)_{*}\left(F^{\prime}\right) .
\end{gathered}
$$

Lemma 6.1. The line bundles $L_{1}, L_{2}, L_{3}$ form a basis of the Picard group of $X$, and the classes $C, E, F$ form a basis of $H_{2}(X, \mathbb{Z})$ (which are all effective).

Proof. We can compute the intersection pairing of these bundles with these curves, which we find to be

\begin{tabular}{c|ccc} 
& $C$ & $F$ & $E$ \\
\hline$L_{1}$ & -1 & -2 & 1 \\
$L_{2}$ & -1 & 1 & 0 \\
$L_{3}$ & 1 & 0 & 0
\end{tabular}

which clearly has determinant -1 . It follows (since $h^{1,1}=3$ ) that the lattices that these generate must be the whole lattice.

We will further need the triple intersections $\Gamma_{i j k}=\int_{X} L_{i} \smile L_{j} \smile L_{k}$, which are computed as follows.

Lemma 6.2. The triple intersections are given by the following.

$$
\begin{array}{llr}
\Gamma_{111}=8 & \Gamma_{112}=-1 & \Gamma_{113}=-2 \\
\Gamma_{122}=-1 & \Gamma_{123}=1 & \Gamma_{133}=0 \\
\Gamma_{222}=0 & \Gamma_{223}=0 & \Gamma_{233}=0 \\
& \Gamma_{333}=0 &
\end{array}
$$

Proof. These are computed simply by restricting the line bundles to the smooth representatives $S, X_{C}, X_{F}$, where the intersections are easy to compute. 
6.1. The rational elliptic surface. The rational elliptic surface $X_{C}$ is realizable as the blowup of $\mathbb{P}^{2}$ at 9 points in general position; as such, its intersection form is $\Gamma_{1,9}$, where $\Gamma_{a, b}$ is the lattice with diagonal intersection form given by

$$
\operatorname{diag}(\underbrace{1, \ldots, 1}_{a}, \underbrace{-1, \ldots,-1}_{b}) .
$$

Let $H, C_{0}, \ldots, C_{8}$ denote the classes of the line and exceptional curves, respectively. Then $3 H-\sum_{i=0}^{8} C_{i}$ and $C_{0}$ span a sublattice which is isomorphic to $\Gamma_{1,1}$ (of discriminant 1), and hence we have a splitting

$$
\Gamma_{1,9} \cong \Gamma_{1,1} \oplus E_{8}
$$

where $E_{8}$ is the unique even, unimodular, negative-definite lattice corresponding to the Dynkin diagram $E_{8}$. We should remark that the classes $C_{0}$ and $3 H-\sum_{i=0}^{8} C_{i}$ are the same as the base and fibre classes $C^{\prime \prime}, E^{\prime \prime}$ of the rational elliptic surface discussed earlier.

Remark 6.1. The canonical divisor on the surface $X_{C}$ is given by

$$
K_{X_{C}}=-3 H+\sum_{i=0}^{8} C_{i}=-E^{\prime \prime}
$$

This allows us now to compute the relationship between the groups $H_{2}\left(X_{C}, \mathbb{Z}\right)$ and $H_{2}(X, \mathbb{Z})$, which we will need later.

Lemma 6.3. The map $H_{2}\left(X_{C}, \mathbb{Z}\right) \rightarrow H_{2}(X, \mathbb{Z})$ is given by

$$
H_{2}\left(X_{C}, \mathbb{Z}\right) \cong \Gamma_{1,1} \oplus E_{8} \stackrel{\text { proj }}{\longrightarrow} \Gamma_{1,1} \subseteq H_{2}(X, \mathbb{Z})
$$

where $\Gamma_{1,1}$ includes via the identification $C:=\left(\iota_{2}\right)_{*} C^{\prime \prime}, E:=\left(\iota_{2}\right)_{*}\left(E^{\prime \prime}\right)$ described earier.

Proof. We first claim that $\left(\iota_{2}\right)_{*} C_{i}=C+E$ for $1 \leq i \leq 8$, and that $\left(\iota_{2}\right)_{*} H=$ $3(C+E)$. This can be seen simply by using the push-pull formula and noting that

$$
\iota_{2}^{*} L_{1}=C_{0} \quad \iota_{2}^{*} L_{2}=-E^{\prime \prime} \quad \iota_{2}^{*} L_{3}=E^{\prime \prime} .
$$

Now, an element $a H+\sum_{i=0}^{8} b_{i} C_{i}$ is in the orthogonal complement of the lattice spanned by $E^{\prime \prime}=3 H-\sum_{i=0}^{8} C_{i}, C^{\prime \prime}=C_{0}$ if and only if

(1) $b_{0}=0$

(2) $3 a+\sum_{i=0}^{8} b_{i}=0$.

Thus, we have that

$$
\begin{aligned}
\left(\iota_{2}\right)_{*}\left(a H+\sum_{i=0}^{8} b_{i} C_{i}\right) & =3 a(C+E)+\sum_{i=0}^{8} b_{i}(C+E) \\
& =\left(3 a+\sum_{i=0}^{8} b_{i}\right)(C+E)=0
\end{aligned}
$$

as claimed.

Finally, we need one fact about effectivity of classes in $H_{2}\left(X_{C}, \mathbb{Z}\right)$.

Lemma 6.4. Let $\beta=C^{\prime \prime}+n E^{\prime \prime}+\lambda \in H_{2}\left(X_{C}, \mathbb{Z}\right)$, where $\lambda \in E_{8}$. Then $\beta$ is effective if and only if $\lambda \cdot \lambda \geq-2 n$. 
Proof. This is a straightforward application of Riemann-Roch. For a divisor $D$ on $X_{C}$, this reads as

$$
\chi(D)=1+\frac{1}{2} D \cdot\left(D-K_{X_{C}}\right) .
$$

In particular, for $D=C^{\prime \prime}+n E^{\prime \prime}+\lambda$, we find that

$$
h^{0}(D)+h^{2}\left(K_{X_{C}}-D\right)=1+n+\frac{1}{2} \lambda \cdot \lambda .
$$

Thus since $K_{X_{C}}-D$ will never be effective, it follows that as long as $n+\frac{1}{2} \lambda \cdot \lambda \geq 0$, that $D$ will have a section, and hence be effective.

6.2. The K3 fibration. To compute the Gopakumar-Vafa invariants of $X$ in the fibre-wise classes (i.e. those which project down to 0 under the map $\pi: X \rightarrow C$ ), we use the machinery of $[8,12$, which we will review here. Moreover, the ideas in this section closely follow the the ideas of [8. For more detail, that article is strongly recommended.

Definition 6.1. Let $\Lambda$ be a rank $r$ lattice. A family of $\Lambda$-polarized K3 surfaces over a base curve $\Sigma$ is a scheme $Z$ over $\Sigma$ together with a collection of line bundles $L_{1}, \ldots, L_{r}$ such that, for each $b \in \Sigma$, the fibre $\left(X_{b},\left.L_{1}\right|_{X_{b}}, \ldots,\left.L_{r}\right|_{X_{b}}\right)$ is a $\Lambda$-polarized K3 surface.

Such a family $Z \stackrel{\pi}{\rightarrow} \Sigma$ yields a map $\iota_{\pi}$ to the moduli space $\mathcal{M}_{\Lambda}$ of $\Lambda$-polarized K3 surfaces. Intersecting the image of the curve with certain divisors in $\mathcal{M}_{\Lambda}$ (see again, [8, 12] ) will produce the Noether-Lefschetz numbers. These are given as follows.

The Noether-Lefschetz divisors consist of those $\Lambda$-polarized K3 surfaces which jump in Picard rank; these are determined by

(1) an integer $h$, such that the square of the new class $\beta$ is $2 h-2$

(2) $r$ integers $d_{1}, \ldots, d_{r}$ which are given by $d_{i}=\int_{\beta} L_{i}$.

We denote such a divisor by $D_{h ; d_{1}, \ldots, d_{r}}$, and we then define

$$
N L_{\left(h ; d_{1}, \ldots, d_{r}\right)}^{\pi}=\int_{\iota_{\pi} \Sigma} D_{h ; d_{1}, \ldots, d_{r}} .
$$

It should be remarked that, by the Hodge index theorem, this will be only be non-zero if the discriminant

$$
\Delta\left(h ; d_{1}, \ldots, d_{r}\right)=(-1)^{r} \operatorname{det}\left(\begin{array}{llll} 
& & & d_{1} \\
& \Lambda & & \vdots \\
& & & d_{r} \\
d_{1} & \cdots & d_{r} & 2 h-2
\end{array}\right)
$$

is non-negative.

Let $r_{0, h}$ denote the reduced Gopakumar-Vafa invariants of a K3 surface in a class $\beta$ such that $\beta \cdot \beta=2 h-2$. From [8], these only depend on the square of $\beta$ (and not its divisibility, as one might expect), and they satisfy the Yau-Zaslow formula (see [1, 4, 8, 13])

$$
\sum_{h=0}^{\infty} r_{0, h} q^{h-1}=\frac{1}{\Delta(q)}=\frac{1}{\eta(q)^{24}}=q^{-1}+24+324 q+3200 q^{2}+\cdots .
$$

It should be remarked that the power of 24 that shows up in this formula is due to the presence of the 24 nodal fibres in our elliptically fibred K3 surfaces. 

by

Let $n_{\left(d_{1}, \ldots, d_{r}\right)}^{Z}$ denote the Gopakumar-Vafa invariants of the threefold $Z$ defined

$$
n_{\left(d_{1}, \ldots, d_{r}\right)}^{Z}=\sum_{\substack{\beta \in H_{2}(Z) \\ \int_{\beta} L_{i}=d_{i}}} n_{\beta}^{Z}
$$

We have the following relation between these invariants.

Theorem 6.5. [12, Theorem $\left.1^{*}\right]$ The invariants $n_{\left(d_{1}, \ldots, d_{r}\right)}^{Z}, r_{0, h}$, and $N L_{h ; d_{1}, \ldots, d_{r}}^{\pi}$ satisfy the following relationship.

$$
n_{\left(d_{1}, \ldots, d_{r}\right)}^{Z}=\sum_{h=0}^{\infty} r_{0, h} N L_{h ; d_{1}, \ldots, d_{r}}^{\pi}
$$

Consider now the restriction of $L_{1}, L_{2}$ to $X_{F}$. We an compute their intersections via Lemma 6.2 to find that we have

$$
\left(\begin{array}{ll}
L_{1} \cdot L_{1} & L_{1} \cdot L_{2} \\
L_{2} \cdot L_{1} & L_{2} \cdot L_{2}
\end{array}\right)=\underbrace{\left(\begin{array}{cc}
-2 & 1 \\
1 & 0
\end{array}\right)}_{\Lambda}
$$

where in this case the rank $r$ of $\Lambda$ is 2 .

What we would like to have is that the triple $\left(X, L_{1}, L_{2}\right)$ is a family of $\Lambda$-polarized K3 surfaces. However, due to the presence of singular fibres (due to the singularities of $\left.\Delta=4 g_{2}^{3}-27 g_{3}^{2}\right)$ this is not the case. However, we can "resolve" this threefold (see [8, 12]) to obtain a threefold $\widetilde{X} \stackrel{\widetilde{\pi}}{\rightarrow} C$ which is such a family. We can then relate the invariants of the two families as follows, allowing us to compute the Gopakumar-Vafa invariants of $X$ as desired.

Lemma 6.6. The invariants of $X, \widetilde{X}$ satisfy

$$
n_{\left(d_{1}, d_{2}\right)}^{\tilde{X}}=2 n_{\left(d_{1}, d_{2}\right)}^{X} .
$$

Our final ingredient is to note that the Noether-Lefschetz numbers are coefficients of a modular form of weight $\frac{22-r}{2}=10$; that is, they are the coefficients of some multiple $E_{4}(z) E_{6}(z)=E_{10}(z)=1-264 q-135432 q^{2}-\cdots$. Thus we need to only compute a single such coefficient to determine all of the Noether-Lefschetz numbers.

Definition 6.2. Let $f(z)=\sum_{n=0}^{\infty} a_{n} z^{n}$. Then we will use the notation

$$
[n] f(z)=a_{n}
$$

to denote the coefficient of $z^{n}$ in $f(z)$.

Using this notation, we have the following lemma.

Lemma 6.7. We have that

$$
N L_{0 ; 0,0}^{\widetilde{X}}=1056
$$

and so consequently we have that

$$
N L_{h ; d_{1}, d_{2}}^{\widetilde{\pi}}=-4\left[\frac{\Delta\left(h ; d_{1}, d_{2}\right)}{2}\right] E_{10}(z) .
$$

Proof. The proof of this is identical to the proofs of Lemma 2 and Proposition 2 of [8], and thus we omit it. 


\section{Computations of the Generating Functions}

We are now ready to compute the generating functions for the Gopakumar-Vafa invariants. The generating functions we are interested are those of the following form.

Choose $\beta=m C^{\prime}+r F^{\prime} \in H_{2}(S)$ (which we will identify from now on for simplicity's sake with its image in $\left.H_{2}(X, \mathbb{Z})\right)$. Define

$$
F_{\beta}(q)=\sum_{n=0}^{\infty} n_{\beta+n E}^{X} q^{n-m-\frac{1}{2} r} .
$$

Remark 7.1. We choose this shift in the exponent of $q$ to match the results in [9]. This ensures that the generating functions that we obtain below will be modular, but we don't have a better interpretation of this shifted power.

We then have the following theorem.

Theorem 7.1. We have the following expressions for generating functions $F_{\beta}(q)$ :

$$
\begin{gathered}
F_{F}(q)=-2 \frac{E_{10}(q)}{\Delta(q)}=-2 q^{-1}+480+282888 q+17058560 q^{2}+, \cdots \\
F_{C}(q)=\frac{E_{4}(q)}{\sqrt{\Delta(q)}}=q^{-\frac{1}{2}}+252 q^{\frac{1}{2}}+5130 q^{\frac{3}{2}}+54760 q^{\frac{5}{2}}+. \cdots
\end{gathered}
$$

Each of these is a meromorphic modular form of weight-2, and moreover each of the generating functions $F_{m F}\left(q^{m}\right)$ is also (meromorphic) modular of the same weight, but for the group $\Gamma_{1}\left(m^{2}\right)$.

The first two of these generating functions are conjectured (with physical justification) in the papers [9, 10, along with a few others. We have not found any prior description of the third, although it is an easy generalization of the first.

We will split the proof of this theorem up into several parts.

Theorem 7.2. We have the equality

$$
F_{F}(q)=-2 \frac{E_{10}(q)}{\Delta(q)} .
$$

Proof. We will compute first the function $F_{F}(q)$. Since we have that the class $F+n E$ is determined uniquely by its integration against $L_{1}, L_{2}$, we have that

$$
n_{F+n E}^{X}=n_{(n-2,1)}^{X}
$$

Combining Lemmata 6.7 6.6, and Theorem 6.5, our generating function $F_{F}(q)$ is given by

$$
\begin{aligned}
F_{F}(q) & =\sum_{n=0}^{\infty} n_{(n-2,1)}^{X} q^{n-1} \\
& =\sum_{n=0}^{\infty} \frac{1}{2} n_{(n-2,1)}^{\widetilde{X}} q^{n-1} \\
& =\frac{1}{2} \sum_{n=0}^{\infty} \sum_{h=0}^{\infty} r_{0, h} N L_{h ; n-2,1}^{\tilde{\pi}} q^{n-1}
\end{aligned}
$$


We can compute the discriminant $\Delta(h ; n-2,1)=2 n-2 h$ which must be nonnegative, so the summation is really over those $n, h \geq 0$ with $n \geq h$. Thus we can write this as

$$
\begin{aligned}
F_{F}(q) & =\frac{1}{2} \sum_{h=0}^{\infty} r_{0, h} \sum_{n=h}^{\infty} N L_{h ; n-2,1}^{\tilde{\pi}} q^{n-1} \\
& =\frac{1}{2} \sum_{h=0}^{\infty} r_{0, h} q^{h-1} \sum_{n=h}^{\infty}(-4)\left[\frac{2 n-2 h}{2}\right] E_{10}(z) q^{n-h} \\
& =-2 \sum_{h=0}^{\infty} r_{0, h} q^{h-1} \sum_{n=h}^{\infty}[n-h] E_{10}(z) q^{n-h} \\
& =-2 \sum_{h=0}^{\infty} r_{0, h} q^{h-1} E_{10}(q) \\
& =-2 \frac{E_{10}(q)}{\Delta(q)} .
\end{aligned}
$$

To prove the next formula, we need the following computation of the GromovWitten invariants (for primitive classes) of a rational elliptic surface.

Theorem 7.3 (1], Theorem 6.2). The generating function for the GopakumarVafa invariants of the rational elliptic surface $X_{C}$ in the classes $C^{\prime \prime}+n E^{\prime \prime}$ is given by

$$
\sum_{n=0}^{\infty} n_{C^{\prime \prime}+n E^{\prime \prime}}^{X_{C}} q^{n-\frac{1}{2}}=\frac{1}{\sqrt{\Delta(q)}}
$$

We now prove the following.

Theorem 7.4. We have the equality

$$
F_{C}(q)=\frac{E_{4}(q)}{\sqrt{\Delta(q)}}
$$

Proof. Recall from Lemma 6.3 that the map $\left(\iota_{2}\right)_{*}: H_{2}\left(X_{C}, \mathbb{Z}\right) \cong \Gamma_{1,1} \oplus E_{8} \rightarrow$ $H_{2}(X, \mathbb{Z})$ is essentially the projection onto the $\Gamma_{1,1}$ factor.

Now, we obtain curves in $X$ in the class $C+n E$ by considering curves in $X_{C}$ in some effective class which pushes forward to this class; from Lemma 6.3 these will be curves of the form $\beta=C^{\prime \prime}+n E^{\prime \prime}+\lambda$ where $\lambda \in E_{8}$. From Lemma 6.4, we know that the effective ones are those with $n \geq-\frac{1}{2} \lambda \cdot \lambda$.

We can now compute that

$$
\begin{aligned}
F_{C}(q) & =\sum_{n=0}^{\infty} n_{C+n E}^{X} q^{n-\frac{1}{2}} \\
& =\sum_{n=0} \sum_{\substack{\left.\beta \in H_{2}\left(X_{C}\right) \\
(\iota 2)\right)_{*} \beta=C+n E}} n_{\beta}^{X_{C}} q^{n-\frac{1}{2}} \\
& =\sum_{n=0}^{\infty} \sum_{\substack{\lambda \in E_{8} \\
-\lambda \cdot \lambda \leq 2 n}} n_{C^{\prime \prime}+n E^{\prime \prime}+\lambda}^{X_{1}} q^{n-\frac{1}{2}} .
\end{aligned}
$$


Now, morally similar to the case of K3 surfaces, from primitive curve classes the invariants $n_{\beta}^{X_{C}}$ only depend on the square of $\beta$. In particular, any such curve can be transformed into one of the form $\beta=C^{\prime \prime}+n E^{\prime \prime}$ by a series of Cremona transformations and permutations of the exceptional classes (see [3]). It follows then that

$$
n_{C^{\prime \prime}+n E^{\prime \prime}+\lambda}^{X_{C}}=n_{C^{\prime \prime}+\left(n+\frac{1}{2} \lambda \cdot \lambda\right) E^{\prime \prime}}^{X_{C}}
$$

and so the generating function becomes

$$
\begin{aligned}
F_{C}(q) & =\sum_{n=0}^{\infty} \sum_{\substack{\lambda \in E_{8} \\
-\lambda \cdot \lambda \leq 2 n}} n_{C_{0}+\left(n+\frac{1}{2} \lambda \cdot \lambda\right) E}^{X_{C}} q^{n-\frac{1}{2}} \\
& =\sum_{\lambda \in E_{8}} q^{-\frac{1}{2} \lambda \cdot \lambda} \sum_{n=-\frac{1}{2} \lambda \cdot \lambda}^{\infty} n_{C_{0}+\left(n+\frac{1}{2} \lambda \cdot \lambda\right) E^{X_{C}}}^{q^{n+\frac{1}{2} \lambda \cdot \lambda-\frac{1}{2}}} \\
& =\sum_{\lambda \in E_{8}} q^{-\frac{1}{2} \lambda \cdot \lambda} \sum_{n=0}^{\infty} n_{C_{0}+n E}^{X_{C}} q^{n-\frac{1}{2}} \\
& =\Theta_{E_{8}}(q)\left(\frac{1}{\sqrt{\Delta(q)}}\right)=\frac{E_{4}(q)}{\sqrt{\Delta(q)}}
\end{aligned}
$$

as claimed (with the last equality being due to the well-known fact that $\Theta_{E_{8}}(q)=$ $\left.E_{4}(q)\right)$.

To prove the last statement in the theorem, we need a little extra notation.

Definition 7.1. Let $f(z)=\sum_{a_{n} z^{n}}$ be a power series, and let $m, k$ be integers with $0 \leq k<m$. We define then

$$
f_{m, k}(z)=\sum_{\substack{n \equiv k \\(\bmod m)}} a_{n} z^{n}=\sum_{n=0}^{\infty} a_{m n+k} z^{m n+k} .
$$

We should note that in the case that $f(z)$ is a modular form of weight $r$ for $S L_{2}(\mathbb{Z})$, then each of the functions $f_{m, k}(z)$ are also modular of the same weight for the subgroup $\Gamma_{1}\left(m^{2}\right)$.

Furthermore, we can expand this definition for values of $k$ outside of the given range by replacing $k$ with a suitable integer congruent to $k(\bmod m)$ within that range. For example, $f_{m,-1}(z)=f_{m, m-1}(z)$.

We can now state more precisely our final theorem.

Theorem 7.5. Let $m>1$. Then the generating function $F_{m F}(q)$ is given by

$$
F_{m F}(q)=\sum_{n=0}^{\infty} n_{m F+n E} q^{n-m}=-2 \sum_{\ell=0}^{m-1}\left(\frac{1}{\Delta(u)}\right)_{m, \ell-1}\left(E_{10}(u)\right)_{m, 1-\ell}
$$

where $q=u^{m}$. 
Proof. This proof follows very similarly to the proof of Theorem 7.2 We similarly begin with noting that $n_{m F+n E}^{X}=n_{(n-2 m, m)}^{X}$, which allows us to write

$$
\begin{aligned}
F_{m F}(q) & =\sum_{n=0}^{\infty} n_{(n-2 m, m)}^{X} q^{n-m} \\
& =\sum_{n=0}^{\infty} \frac{1}{2} n_{(n-2 m, m)}^{\widetilde{X}} q^{n-m} \\
& =\frac{1}{2} \sum_{n=0}^{\infty} \sum_{h=0}^{\infty} r_{0, h} N L_{h ; n-2 m, m}^{\widetilde{\pi}} q^{n-m} .
\end{aligned}
$$

In this case, the discriminant $\Delta(h ; n-2 m, m)=2-2 h+2 n m-2 m^{2}$ which as usual must be non-negative, leaving us summing over all pairs $(h, n)$ such that $n \geq m+\frac{h-1}{m}$. Thus we obtain

$$
F_{m F}(q)=-2 \sum_{h=0}^{\infty} r_{0, h} \sum_{n \geq m+\frac{h-1}{m}}\left[1-h+n m-m^{2}\right] E_{10}(z) q^{n-m} .
$$

To simplify this further, we split the summation over $h$ into a sum over congruence classes mod $m$. If we let $q=u^{m}$, then this yields the following.

$$
\begin{aligned}
F_{m F}(q) & =-2 \sum_{\ell=0}^{m-1} \sum_{h=0}^{\infty} r_{0, m h+\ell} \sum_{n \geq m+h+\frac{\ell-1}{m}}[1-\ell+m(n-h-m)] E_{10}(z) u^{n m-m^{2}} \\
& =-2 \sum_{\ell=0}^{m-1} \sum_{h=0}^{\infty} r_{0, m h+\ell} u^{m h+\ell-1} \sum_{n \geq m+h+\frac{\ell-1}{m}}[1-\ell+m(n-h-m)] E_{10}(z) u^{m(n-h-m)-\ell+1} \\
& =-2 \sum_{\ell=0}^{m-1} \sum_{h=0}^{\infty} r_{0, m h+\ell} u^{m h+\ell-1}\left(E_{10}\right)_{m, 1-\ell}(u) \\
& =-2 \sum_{\ell=0}^{m-1}\left(\frac{1}{\Delta(u)}\right)_{m, \ell-1}\left(E_{10}(u)\right)_{m, 1-\ell}
\end{aligned}
$$

which ends the proof.

The above results show that we end up with meromorphic modular forms when we consider generating functions for Gopakumar-Vafa invariants for curve classes of the form $m F+n E$ and $C+n E$. From the conjectured results in [9], it seems that we should end up with similar results for curve classes of the form $r C+n E$; a natural approach to study these would be to use the recursion of $[3$, which we will look to do at a future date.

\section{REFERENCES}

1. Jim Bryan and Naichung Conan Leung, The enumerative geometry of K3 surfaces and modular forms, J. Amer. Math. Soc. 13 (2000), no. 2, 371-410 (electronic). MR MR1750955 (2001i:14071)

2. R. Gopakumar and C. Vafa, M-theory and topological strings I and II, 1998.

3. L. Göttsche and R. Pandharipande, The quantum cohomology of blow-ups of $\mathbf{P}^{2}$ and enumerative geometry, J. Differential Geom. 48 (1998), no. 1, 61-90. MR 1622601 (99d:14057)

4. Lothar Göttsche, A conjectural generating function for numbers of curves on surfaces, Comm. Math. Phys. 196 (1998), no. 3, 523-533. MR 1645204 (2000f:14085) 
5. Friedrich Hirzebruch, Classical algebraic geometry and Calabi-Yau manifolds, Calabi conference at University of Wisconsin, 2003.

6. Klaus Hulek and Remke Kloosterman, Calculating the Mordell-Weil rank of elliptic threefolds and the cohomology of singular hypersurfaces, Ann. Inst. Fourier (Grenoble) 61 (2011), no. 3, 1133-1179. MR 2918726

7. Sheldon Katz, Gromov-Witten, Gopakumar-Vafa, and Donaldson-Thomas invariants of Calabi-Yau threefolds, Snowbird lectures on string geometry, Contemp. Math., vol. 401, Amer. Math. Soc., Providence, RI, 2006, pp. 43-52. MR 2222528 (2007b:14121)

8. A. Klemm, D. Maulik, R. Pandharipande, and E. Scheidegger, Noether-Lefschetz theory and the Yau-Zaslow conjecture, J. Amer. Math. Soc. 23 (2010), no. 4, 1013-1040. MR 2669707 (2011j:14121)

9. Albrecht Klemm, Jan Manschot, and Thomas Wotschke, Quantum geometry of elliptic CalabiYau manifolds, Commun. Number Theory Phys. 6 (2012), no. 4, 849-917. MR 3068410

10. Albrecht Klemm, Peter Mayr, and Cumrun Vafa, BPS states of exceptional non-critical strings, Nuclear Phys. B Proc. Suppl. 58 (1997), 177-194, Advanced quantum field theory (La Londe les Maures, 1996). MR 1486340 (99a:81145)

11. Yuri Manin, Cubic forms: Algebra, geometry, arithmetic, North-Holland Mathematical Library, Elsevier Science, 1986.

12. D. Maulik and R. Pandharipande, Gromov-Witten theory and Noether-Lefschetz theory, A Celebration of Algebraic Geometry (B. Hassett, J. McKernan, J. Starr, and R. Vakil, eds.), vol. 18, Clay Math Proc., 2013, pp. 469-508.

13. Shing-Tung Yau and Eric Zaslow, BPS states, string duality, and nodal curves on K3, Nuclear Phys. B 471 (1996), no. 3, 503-512. MR MR1398633 (97e:14066)

Department of Mathematics and Statistics, Queen's University, Kingston, Ontario CANADA K7L 3N6

E-mail address: simon@mast.queensu.ca; yui@mast.queensu.ca 